\title{
Проблемы развития отрасли оптико-электронного приборостроения
}

Ю.Г. Якушенков, М. В. Хорошев М.В., mkhoroshev@miigaik.ru, Московский государственный университет геодезии и картографии (МИИГАиК), www.miigaik.ru, Москва, Россия

В статье рассмотрены проблемы обеспечения развития оптико-электронного приборостроения в нашей стране. Показаны основные направления и задачи развития современного оптического приборостроения и факторы, препятствующие их реализации. Предложены меры для устранения или снижения влияния этих факторов. Описана методика подготовки специалистов в области оптотехники и лазерных технологий в МИИГАиК.

Ключевые слова: оптико-электронные системы и комплексы, фотоника, оптотехника, кадровое обеспечение, инженерное образование, Комитет по экономической политике, промышленности, инновационному развитию и предпринимательству Государственной Думы

\section{Problems of Development of the Optical and Electronic Instrument Industry}

Yu.G.Yakushenkov, M.V.Khoroshev M.V., mkhoroshev@miigaik.ru,

Moscow State University of Geodesy and Cartography (MIICAiK), www.miigaik.ru, Moscow, Russia

The article considers the problems of ensuring the development of optoelectronic instrument engineering in our country. The main directions and tasks of the development of modern optical instrumentation and the factors impeding their implementation are shown. Actions are proposed to eliminate or reduce the influence of these factors. The methodology of training specialists in the field of optics and laser technologies at MIIGAiK is described.

Keywords: optical electronic systems and complexes, photonics, optotechnics, staffing, engineering education, Committee on Economic Policy, Industry, Innovative Development and Entrepreneurship of the State Duma

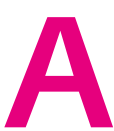
вторы не претендуют на всесторонний анализ состояния дел в области оптикоэлектронного приборостроения на данный момент и общую оценку всех направлений и возможностей фотоники в нашей стране. Главное

\footnotetext{
Примечание редактора: Статья рекомендована программным комитетом конференции, проходившей в рамках специализированной выставки лазерной, оптической и оптоэлектронной техники «Фотоника. Мир лазеров и оптики. 2019».
}

задача - это акцентировать внимание на проблемах, которые тормозят нормальное развитие оптико-электронного приборостроения в приложениях, наиболее близких к деятельности авторов.

Основными направлениями развития современного оптико-электронного приборостроения, по нашему мнению, являются:

1. развитие общей теории и методов проектирования оптико-электронных приборов (ОЭП) и оптико-электронных систем (ОЭС); 
2. совершенствование методов расчета, проектирования и технологии изготовления отдельных узлов и ОЭС в целом, позволяющих повысить чувствительность, увеличить разрешающую способность, быстродействие, срок службы, надежность, снизить энергопотребление, уменьшить габариты и стоимость этих приборов и систем;

3. совершенствование элементной базы: оптических материалов и систем, источников и приемников излучения, электронных компонентов, включая микропроцессоры, дисплеи, электроприводы и тому подобные детали. Конечной целью такого совершенствования является увеличение дальности действия оптикоэлектронных приборов и систем (ОЭПиС) и точности измерения или слежения за наблюдаемыми объектами, повышение вероятности обнаружения объектов на фоне маскирующих их помех и другие показатели качества работы этих приборов и систем;

4. освоение новых спектральных диапазонов и/или их совмещение, т.е. создание приборов, одновременно работающих в двух и более диапазонах или работающих на длинах волн, относящихся к видимому и двум участкам ИК-диапазона;

5. создание многодиапазонных матричных приемников излучения;

6. ввод в состав ОЭП современных микропроцессоров и интегральных схем;

7. разработка адаптивных ОЭП и ОЭС, т.е. приборов и систем, автоматически "приспосабливающихся" $\mathrm{K}$ изменяющимся условиям их работы, например приборов, у которых величина просматриваемого пространства (поле) меняется, а чувствительность приемника излучения "загрубляется", если мощность поступающего на него оптического сигнала увеличивается, или повышается, если эта мощность уменьшается;

8. совершенствование технологии изготовления и сборки отдельных деталей и узлов и систем в целом, а также методов их испытаний и исследований;

9. разработка многофункциональных систем, например предназначенных как для обнаружения каких-либо излучателей на фоне помех, так и для измерения их координат или автоматического слежения за ними;
10. создание комплексной аппаратуры, включающей в свой состав оптико-электронные, радиоэлектронные, акустические и другие системы и приборы;

11. развитие "двойных технологий".

Анализируя вышеперечисленные направления развития, обнаруживаешь очевидное - для их реализации должна быть достигнута координация между деятельностью производственных, научных и учебных организаций в деле решения технических и кадровых вопросов современного обеспечения оптико-электронного приборостроения.

Как известно, сегодня различают три поколения ОЭс. К первому относятся системы с одноплощадочным приемником излучения, работающие в одном спектральном диапазоне. Ко второму системы с многоэлементным фотоприемными устройствами (ФПУ) в виде линейки или матрицы отдельных фотоприемников, работающих также в одном спектральном диапазоне. К третьему поколению ОЭС относят системы с многоэлементным матричным ФПУ, работающим в двух или более спектральных диапазонах. В системах 1-го и 2-го поколений просмотр поля обзора (сцены) осуществляется путем взаимного перемещения вдоль одной или двух ортогональных координат изображения сцены и ФПУ с помощью оптикомеханического сканирования. В ОЭС 2-го поколения с матричным ФПУ и в ОЭС 3-го поколения просмотр изображения сцены производится путем снятия сигналов с отдельных чувствительных элементов матричного ФПУ. Применение двух- и многодиапазонных матричных ФПУ, работающих в двух- или более спектральных диапазонах, позволяет упростить оптико-механическую схему современных ОЭС, заметно уменьшить их габариты, массу и энергопотребление, увеличить быстродействие.

Наряду с такими системами, в настоящее время успешно функционируют двух ${ }^{-}$и многоканальные ОЭС, в которых разделение на отдельные спектральные каналы происходит в оптической системе. Иногда к ОЭС 3-го поколения относят системы, в которых один из спектральных каналов работает в видимой области спектра, а остальные - в ИК-области. В ряде систем 3-го поколения один спектральный диапазон, например длинноволновый ИК-диапазон (8-14 мкм), используется преимущественно для обнаружения целей, а другой, например средневолновый ИК-диапазон (3-5 мкм), - для идентификации объектов цели и их измерений. 
В современных ОЭС 3-го поколения используются современные достижения цифровых методов обработки изображений. Там нашли практическое применение цифровые системы сложения изображений, образующихся в отдельных рабочих спектральных каналах. При этом проще осуществляются регулировка яркости и выделение отдельных целей, устраняются различия по времени полученных изображений, сформированных в отдельных каналах, обеспечивается большее угловое поле при независимой работе спектральных каналов. Кроме того, цифровое изображение легче встраивается в общий комплекс наблюдения и управления операциями.

В качестве примеров важнейших областей применения современных ОЭС 3-го поколения укажем следующие:

- круглосуточные обнаружение и целеуказание в любую погоду;

- поиск движущихся и неподвижных объектов на больших площадях и определение их координат;

- целеуказание и передача данных о координатах целей другим системам и операторам;

- автоматическое сопровождение движущихся целей;

- автоматическая обработка сигналов для выдачи привязанной к карте информации на экран оператора и в систему автоматического обнаружения объектов;

- обнаружение и классификация химических и биологических веществ.

В качестве иллюстрации возможностей современных высокоточных информационноизмерительных ОЭС рассмотрим данные о погрешностях, которые они сегодня обеспечивают:

- погрешности измерения малых линейных величин - десятые доли микрометра;

- погрешности измерения больших линейных величин, достигающих нескольких километров - единицы милиметров;

- погрешности измерения малых угловых величин, не превышающих нескольких градусов - десятые доли угловой секунды;

- погрешности измерения больших угловых величин, достигающих нескольких десятков градусов - единицы угловых секунд;

- погрешности измерения относительно невы ${ }^{-}$ соких температур или их перепадов (в диапазоне 100-400 K) - до нескольких мК;

- погрешности измерения промежутков времени - до $10^{-12} \mathrm{c}$;
- погрешности измерения спектральных величин (длин волн) - до 0,1 мкм.

Как видно из вышеперечисленного, в значительной степени развитие новых направлений и приложений оптико-электронного приборостроения зависит от доступности к широкому спектру элементной базы, обеспечивающей возможность появления и решения новых теоретических и схемотехнических предложений. В подавляющем большинстве оптико-электронных приборов и систем элементная база либо напрямую поставляется из-за рубежа, либо поступает в страну через дилеров с российской аккредитацией. K сожалению, отечественная продукция такого же типа, как правило, не конкурентоспособна с зарубежной. Это резко ограничивает развитие отечественной техники. Многим знакомы трудности выполнения государственного оборонного заказа даже по чисто формальным признакам. Очевидно, что решить эту задачу можно только совместными усилиями науки и производства. Но кто будет координировать эти действия? Приходится только сожалеть об отсутствии современного Государственного комитета по науке и технологиям (ГКНТ) или какого-либо иного посредника, призванного объединить усилия организаций разных ведомств. Плюс к этому вызывает сожаление и отсутствие потребности в подготовке соответствующих кадров. Добавим к сказанному отсутствие правовой базы взаимодействия организаций различных форм собственности, принадлежащих разным учредителям. В настоящий момент времени предприятия оптической промышленности обновляют оборудование и технологии в основном за счет финан ${ }^{-}$ совых кредитов. Такие условия исключают у них стимулы финансирования целевой подготовки кадров.

Рассмотрим задачи, стоящие в ближайшие годы перед разработчиками ОЭС 3-го поколения:

- разработка методики выбора оптимальных спектральных рабочих диапазонов для систем конкретного назначения;

- определение корреляционных связей между сигналами, получаемыми в различных спектральных диапазонах;

- классификация различных объектов (материалов, покрытий, образований), обнаруживаемых или наблюдаемых с помощью ОЭС;

- использование различий в поляризационных характеристиках отдельных объектов и фонов, на которых они наблюдаются;

- совершенствование элементной базы, в частности создание новых многодиапазонных 
(многоспектральных) ФПУ высокого пространственного разрешения; оптических систем, работающих в широком спектральном диапазоне; снижение массогабаритных параметров и энергопотребления; уменьшение стоимости компонентов ОЭС и систем в целом и т.д.;

- создание адаптивных ОЭС 3-го поколения;

- совершенствование методов проектирования и испытаний ОЭС, в частности дальнейшее развитие компьютерного моделирования самих систем и условий их эксплуатации.

Однако эти задачи не могут быть эффективно решены, пока отсутствует нормативная база в области фотоники, лазерной техники и лазерной безопасности. Последнюю пару лет Технический комитет по стандартизации № 296 "Оптика и фотоника" Росстандарта начал проявлять свою активность, и мы ждем реальных результатов. Но это всего лишь первые шаги, и они не всегда качественные. Тому пример недавняя попытка согласования очередного проекта стандарта «Фотоника. Основные определения", на содержание которого специалисты дали существенные замечания.

Кроме того, для практической реализации решения этих задач требуется материальное обеспечение, а главное - наличие подготовленных кадров для проектирования, исследований и создания высококачественных ОЭС. Таким образом, на первый план опять выступают вопросы подготовки кадров.

Подготовка специалистов в области оптотехники и лазерного приборостроения с точки зрения использования информатики и компьютерных технологий может быть разделена на три этапа:

1. изучение общих принципов информатики и компьютерных технологий;

2. обучение использованию этих технологий при проектировании типовых блоков оптических и оптико-электронных систем;

3. обучение методологии использования компьютерного моделирования при системном подходе $\mathrm{K}$ проектированию оптикоэлектронной системы как единого целого.

Сегодня роль информационных и компьютерных технологий, в частности, компьютерного моделирования, при подготовке специалистов общеизвестна. Большое внимание им уделяется при реализации образовательных программ в области оптотехники и лазерного приборостроения. Сложность современных оптико-электронных приборов и комплексов, состоящих из разнородных по физической природе блоков и узлов (оптических, механических, электронных и др.), требует на всех этапах их проектирования, изготовления, испытаний и исследований использовать системный подход, который сегодня невозможен без использования компьютерных технологий.

Первый этап реализуется в многочисленных учебных планах и программах подготовки будущих специалистов достаточно давно. Например, изучаются курсы "Информатика", "Математическое моделирование", "Инженерная и компьютерная графика". Программы этих дисциплин постоянно корректируются и актуализируются, совершенствуется их аппаратно-программное обеспечение.

Второй этап реализуется при изучении отдельных общепрофессиональных дисциплин, как "Прикладная оптика", "Источники и приемники оптического излучения", "Электроника и микропроцессорная техника", "Основы конструирования точных приборов", "Расчет оптических систем", "Дизайн оптических приборов" и др. При этом используются современные компьютерные методы синтеза, анализа и оптимизации типовых блоков оптических и оптико-электронных приборов и систем.

Следует отдельно указать важность компьютерных технологий, учитывая, что именно специалист в области оптотехники и оптико-электронных приборов и комплексов является первым, кто должен оптимизировать вид сигналов, поступающих от системы первичной обработки информации (СПОИ) на вход электронного блока. Возможности современной вычислительной техники обуславливают высокие требования к параметрам и характеристикам блоков и узлов, составляющих основу большинства современных информационноизмерительных и следящих СПОИ - оптических систем, анализаторов изображения, фотоприемных устройств. Недостаток внимания к оптимизации СПОИ приводит к тому, что процесс проектирования может выполняться лишь на системотехническом и схемотехническом (структурном и алгоритмическом) уровнях, но не на параметрическом. Это означает, что образовательный процесс не приводит к овладению умениями и навыками по выбору конкретных числовых значений параметров и характеристик как всей системы, так и ее отдельных узлов.

В МИИГАиКе на этом этапе используются компьютерные программы для контроля, тестирования и оценки усвоения общепрофессиональных компетенций обучающихся. Тестирование концентрирует внимание студентов на основных разделах дисциплины, а также на некоторых вопро- 
сах, практически важных для оценки показателей качества работы отдельных узлов и блоков типо вых оптических и оптико-электронных приборов и систем. Оно также помогает выявить недостатки в усвоении отдельных разделов курса. Уже на этом этапе система тестов может быть разделена на две группы - для внутридисциплинарного и междисциплинарного (комплексные тесты и контрольные задания) контроля. Первая служит для проверки усвоения основных теоретических положений и привития навыков обращения с ними только для одной конкретной дисциплины. При этом проверяется знание системы основных параметров и характеристик одного из блоков всей системы и важнейших их значений, формул для их качественного и количественного описания. Междисциплинарные тесты и контрольные задания играют особую роль при подготовке будущего специалиста. Студент должен знать как основные физические принципы их функционирования, так и методы их сочетания в рамках единой конструкции. Он должен уметь описывать конкретные ситуации и их компьютерные модели (субмодели) для типовых отдельных систем. Здесь необходимо опираться на опыт аналогичных разработок, действующие стандарты и каталоги существующей элементной базы.

На третьем этапе очень важно использовать знания, умения и навыки, приобретенные на втором этапе, для использования их в рамках освоения обобщенной методологии компьютерного моделирования разрабатываемых оптико-электронных систем и комплексов. Эта методология позволяет решить многие проблемы схемотехнического и параметрического анализа или синтеза, часто возникающие, по крайней мере, на первых этапах разработки разнообразных современных оптических и оптико-электронных приборов и систем, работающих в сложных и меняющихся условиях эксплуатации. Компьютерное моделирование, используемое при проектировании, исследованиях и испытаниях современных приборов и систем, позволяет моделировать различные их структуры с требуемой адекватностью, вычислять значения показателей эффективности их работы (показатели качества) при разнообразных фоноцелевых обстановках, оценивать полноту и совершенство существующей элементной базы, решать другие задачи, например, сокращать время разработки и его стоимость. В ряде случаев оно заметно сокращает объем дорогостоящих и длительных экспериментов.

Описанная выше методика подготовки кадров достаточно характерна для всех вузов, ведущих подготовку в области фотоники и ее технологий, отличаясь в деталях в зависимости от возможностей каждого учебного заведения. При этом следует отметить явное ослабление внимания к подготовке кадров различного уровня в нашей области науки и техники. И вопрос не только в том, что фотонику до сих пор не включили в перечень критических технологий ${ }^{*}$ и национальных проектов.

Вузы формально не занимаются профессиональной подготовкой специалистов, а оказывают образовательные услуги, оставляя уровень получаемых знаний для профессии на совести преподавателя. Идет заметное сокращение бюджетных мест для подготовки специалистов с высшим образованием для оптотехники, лазерной техники и лазерных технологий. Ограничиваются возможности предприятий в выделении средств на подготовку кадров при разработке новой техники и технологий. Возможности обновления материального обеспечения учебного процесса как по бюджетной линии, так и по хоздоговорной тематике весьма ограничены, а потому снижается качество подготовки требуемых специалистов. Не стоит забывать, что подготовку в области фотоники ведут не только национальные университеты с усиленным финансированием, но и региональные вузы, где помощь местной промышленности в подготовке кадров жизненно необходима. Статистика показывает, что 2/3 предприятий в области фотоники относятся к малым или средним, для которых правовая возможность выделять средства на обучение без ущерба для своего существования отсутствует. Без создания нужной мотивации для всех участников процесса разработки и изготовления новых образцов оптико-электронных приборов добиться эффективных результатов, так называемых прорывов, будет очень сложно, а может быть, даже невозможно.

Нельзя не сказать и еще об одной болевой точке как высшей школы, так и реальных производств. Речь идет об уровне преподавания дисциплин физики и химии в средней школе. Все вузы вынуждены при обучении студентов тратить

Перечень критических технологий - один из основ ных инструментов государственной политики Российской Федерации в области развития отечественной науки и технологий; национальные проекты направлены на обеспечение прорывного научно-технологического и социально-экономического развития России, повышения уровня жизни, создания условий и возможностей для самореализации и раскрытия таланта каждого человека; http://government.ru. 


\section{Юрий Григорьевич Якушенков}

Дорогие читатели. В этом номере журнала ФОТОНИКА на второй полосе в последний раз в ряду членов его редакционного совета будет стоять имя Юрия Григорьевича Якушенкова, заслуженного деятеля науки и техники РСФСР, профессора, доктора технических наук. Мы еще не раз встретимся с его творческим наследием на страницах нашего журнала, но больше не услышим его бодрого и уверенного голоса, не встретим его на оптических выставках.

Оптика занимала в его жизни важное место. Юрий Григорьевич любил повторять, что, как бы ни назывались разделы науки "Оптика", как бы ни пересекалось их внутреннее содержание, все они базируются на фундаментальных началах современной физики. Он окончил с золотой медалью московскую школу, поступил на оптический факультет МИИГАиК, с отличием окончил его в 1960 году. В 1963 году защитил кандидатскую диссертацию, а уже через пять лет вышел на защиту докторской. Юрий Григорьевич стал одним из первых организаторов подготовки в нашей стране высококвалифицированных разработчиков оптико-электронных приборов. Его учебник "Теория и расчет оптико-электронных приборов" переиздавался шесть раз. В 1972 году он возглавил кафедру оптико-электронных приборов, с 1985 - факультет оптического приборостроения МИИГАиК. Ю. Г. Якушенков деятельно

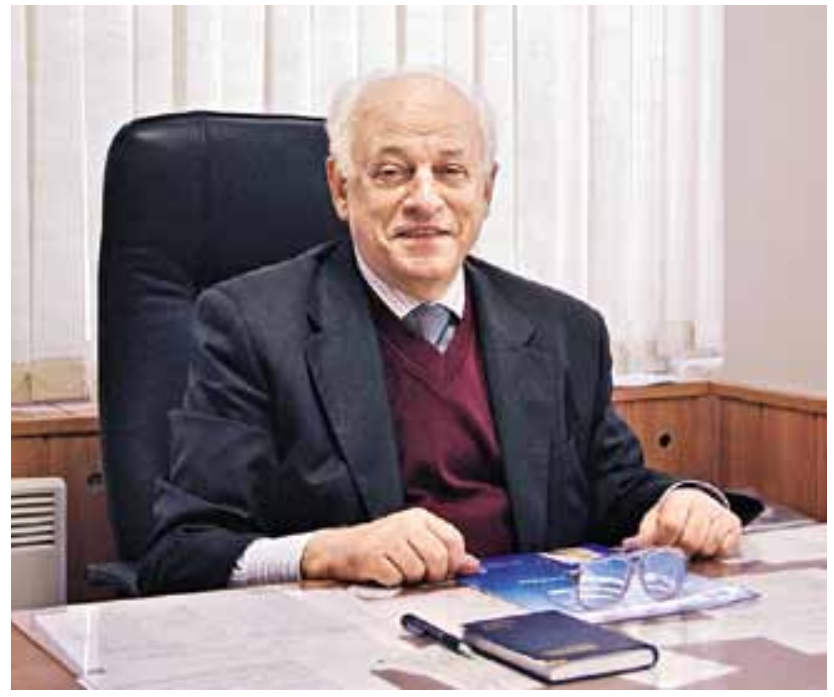

укреплял связи системы высшего образования с наукой и промышленностью. Он постоянно участвовал в организации международных и российских форумов и конференций. Благодаря его авторитетному мнению в стране были поддержаны многие научно-технические проекты по созданию оптико-электронных обзорных и поисковых систем, во многом благодаря его имени развивался и приобрел известность и наш журнал.

Теперь ученики Юрия Григорьевича продолжат его дело и передадут новому поколению инженеров-оптиков уверенность в том, что оптика определяет прогресс в индустрии, в космических исследованиях, в системах передачи связи, в гражданской и военной технике. Редакция журнаяа ФОТОНИКА

значительные объемы занятий на ликвидацию заметной безграмотности абитуриентов в базовых дисциплинах, без которых техническое образование невозможно.

Практически все вышеперечисленные проблемы вызваны недостаточным финансированием или отсутствием правовой базы для его осуществления за счет различных источников. Очевидно, что государственные деятели должны понимать важность нашей отрасли для развития экономики и обороноспособности страны и направить свои действия на обеспечение бюджетной подготовки нужного количества специалистов требуемого профиля на уровне базового образования. Специализированное или целевое образование должно осуществляться за счет средств кон- кретных хозяйствующих субъектов - заказчиков. Надо согласовывать с заказчиками учебные программы, а предприятия, расходующие свои средства на подготовку кадров, необходимо финансово поощрять.

Для этого необходимо разработать соответствующие законодательные условия. Надеемся, что депутаты Государственной Думы из Комитета по экономической политике, промышленности, инновационному развитию и предпринимательству, объединившиеся вокруг Экспертного совета по фотонике, направят свою деятельность на решение проблем оптико-электронной индустрии. Логика развития техники и технологий выводит фотонику на сцену инновационного развития нашей страны, и не замечать этого нельзя. 